\title{
Distributed computation of generalized Nash equilibria in quadratic aggregative games with affine coupling constraints
}

\author{
Dario Paccagnan, Basilio Gentile, Francesca Parise, Maryam Kamgarpour and John Lygeros
}

\begin{abstract}
We analyse deterministic aggregative games, with large but finite number of players, that are subject to both local and coupling constraints. Firstly, we derive sufficient conditions for the existence of a generalized Nash equilibrium, by using the theory of variational inequalities together with the specific structure of the objective functions and constraints. Secondly, we present a coordination scheme, belonging to the class of asymmetric projection algorithms, and we prove that it converges R-linearly to a generalized Nash equilibrium. To this end, we extend the available results on asymmetric projection algorithms to our setting. Finally, we show that the proposed scheme can be implemented in a decentralized fashion and it is suitable for the analysis of large populations. Our theoretical results are applied to the problem of charging a fleet of plug-in electric vehicles, in the presence of capacity constraints coupling the individual demands.
\end{abstract}

\section{INTRODUCTION}

Recent years have witnessed an increasing interest in the analysis of complex systems, comprising interconnected agents [1]. A main challenge for such systems is the development of control algorithms that take into account the individual objective of each agent, their local and global constraints and, at the same time, require minimum exchange of information among the agents and a central coordinator.

In this paper, we focus on the specific class of noncooperative games in which the objective of each agent depends on the other players only via an aggregate quantity of the agents' decisions. Specific examples include traffic or wireless transmission networks where travel times or transmission rates depend on the overall congestion level [2], [3] and Cournot electricity markets where the electricity price depends on the aggregate demand [4]. This additional structure is formalized in the framework of aggregative games [5] and can be exploited to analyse systems with a large numbers of agents, referred to as a population. Distributed algorithms that guarantee convergence to a Nash equilibrium in aggregative games have been recently proposed in the literature, under various assumptions on the agents' information and communication structures. Specifically, [6], [7] suggest the use of gradient based schemes to coordinate the strategies of the agents to a Nash equilibrium in games with finite population size, nonlinear cost functions and local constraints. In [8], [9] the use of broadcast signals, to which agents

The first three authors contributed equally. This work was supported by the Swiss Nano-Tera project HeatReserves, Swiss Competence Centers for Energy Research FEEB\&D, by the European Commission under project DYMASOS (FP7-ICT 611281) and by the Swiss National Science Foundation (grant 2-773337-12). D. Paccagnan, B. Gentile, F. Parise, M. Kamgarpour and J. Lygeros are with the Automatic Control Laboratory, ETH Zürich, Switzerland. Email: \{dariop, gentileb, parisef, mkamgar, lygeros\}econtrol.ee.ethz.ch. react optimally, is suggested for games with quadratic cost functions and local constraints. Convergence to an $\varepsilon$-Nash equilibrium is guaranteed, where $\varepsilon$ approaches zero as the number of agents becomes large. Similar asymptotic results are common in the literature of mean field game theory. The latter focus on unconstrained stochastic aggregative games with linear dynamics and quadratic cost functions [10], as well as with generic cost functions [11].

All the aforementioned works do not consider coupling between the decisions of different agents. These however arise in many important applications, such as electricity markets, in which agents must satisfy grid and operational constraints, or congestion games, in which agents share a common infrastructure. Since the seminal work of [12], a number of results have been derived for the analysis [13] and control [14] of generalized Nash equilibria (i.e., Nash equilibria for games with coupling constraint) in the case of generic non cooperative games. Attention has been devoted to the characterization of a specific type of generalized Nash equilibria called variational equilibria [15]. The aim of the paper is twofold. First, we specialize the variational equilibria existence results to the important case of deterministic aggregative games. Second, we propose decentralized algorithms and show their convergence for any population size and in the presence of both local and coupling constraints. The analysis is based on the theory of Variational Inequalities (VI). As such, we focus on aggregative games characterized by quadratic cost functions and linear coupling constraints. This choice allows us to use an algorithm belonging to the class of Asymmetric Projection Algorithms (APAs) [13]. Specifically, we show that by cleverly choosing the design parameter the suggested APA can be implemented in a decentralized fashion and we derive conditions that guarantee its convergence. To this end, we extend the available literature results on convergence of APAs to a new class of affine VIs. We then provide an insight between the proposed method and alternative distributed optimization algorithms applicable to our problem formulation, under the additional assumption that the game is potential [16]. Finally, to illustrate our theoretical results, we apply our methodology to the well-studied problem of charging a fleet of Plug-in Electric Vehicles (PEVs) [6], [17]. In contrast to past work we can include coupling constraints, arising for example from capacity limits.

The paper is organized as follows. In Section II we introduce linear quadratic aggregative games with coupling constraints. In Section III we derive conditions on existence of generalized Nash equilibria and uniqueness of the vari- 
ational equilibrium. In Section IV we propose a distributed algorithm to find the variational equilibria. In Section $\mathrm{V}$ we discuss potential games. In section VI we analyse the PEVs charging problem.

Notation: The standard inner product on $\mathbb{R}^{n}$ is denoted by $\langle\cdot, \cdot\rangle: \mathbb{R}^{n} \times \mathbb{R}^{n} \rightarrow \mathbb{R} ; I_{n}$ the $n$-dimensional identity matrix and $\mathbb{1}_{n}$ the vector of unit entries. Given $A \in \mathbb{R}^{n \times n}$ not necessarily symmetric, $A \succ 0(\succeq 0) \Leftrightarrow x^{\top} A x>0(\geq 0)$, $\forall x \neq 0 ; A_{s}:=\frac{A+A^{\top}}{2} . A \otimes B$ denotes the Kronecker product. Given $N$ vectors $x^{i} \in \mathbb{R}^{n},\left[x^{1} ; \ldots ; x^{N}\right]:=\left[x^{i}\right]_{i=1}^{N}:=$ $\left[x^{1^{\top}}, \ldots, x^{N^{\top}}\right]^{\top} ; x_{-i}:=\left[x_{1}, \ldots, x_{i-1}, x_{i+1}, \ldots, x_{N}\right] \in$ $\mathbb{R}^{n(N-1)}$; Given $A \in \mathbb{R}^{m \times N n}$, we define $A_{(:, i)} \in \mathbb{R}^{m \times n}$ such that $A=\left[A_{(:, 1)}, \ldots, A_{(:, N)}\right]$. Given a function $g(x): \mathbb{R}^{n} \rightarrow$ $\mathbb{R}^{m}$ we define the matrix $\nabla_{x} g(x) \in \mathbb{R}^{n \times m}$ componentwise as $\left[\nabla_{x} g(x)\right]_{i, j}:=\frac{\partial g_{j}(x)}{\partial x^{i}}$. Given $N$ matrices $\left\{C^{i} \in\right.$ $\left.\mathbb{R}^{n \times n}\right\}_{i=1}^{N}, \operatorname{diag}\left(C^{i}\right) \in \mathbb{R}^{N n \times N n}$ is a block diagonal matrix with $C^{i}$ as blocks. $\mathcal{U}[a, b]$ represents the uniform distribution on the real interval $[a, b]$.

\section{PROBLEM FORMULATION}

Let us consider a single shot aggregative game characterized by a population of $N$ agents. Each agent $i$ aims at minimizing a quadratic cost function $J^{i}\left(x^{i}, x^{-i}\right): \mathbb{R}^{N n} \rightarrow \mathbb{R}$ depending on its own strategy and on the average strategy

$$
J^{i}\left(x^{i}, x^{-i}\right):=x^{i \top} Q^{i} x^{i}+\left(C^{i} \sigma(x)+c^{i}\right)^{\top} x^{i},
$$

where $Q^{i} \in \mathbb{R}^{n \times n}, C^{i} \in \mathbb{R}^{n \times n}, c^{i} \in \mathbb{R}^{n}$ and $\sigma(x):=$ $\frac{1}{N} \sum_{j=1}^{N} x^{j}$. Note that the cost of each agent depends on the other players' strategies only via the interaction function $f^{i}(\sigma(x)):=C^{i} \sigma(x)+c^{i}$. This type of dependence arises in all those applications where $x^{i}$ denotes the usage level of a commodity whose per unit cost $f^{i}(\cdot)$ depends on the average usage level of the whole population. We assume that the decision variable of the $i$-th player is locally constrained to $x^{i} \in \mathcal{X}^{i} \subset \mathbb{R}^{n}$, and that the stacked vector of strategies, $x=\left[x^{1} ; \ldots ; x^{N}\right]$, has to satisfy $m$ linear global constraints

$$
x \in \mathcal{C}:=\left\{x \in \mathbb{R}^{N n} \mid A x \leq b\right\} \subset \mathbb{R}^{N n},
$$

with $A \in \mathbb{R}^{m \times N n}, b \in \mathbb{R}^{m}$. These constraints could model, for example, the fact that each agent has a feasible range for its usage level and the overall usage level cannot exceed a global capacity constraint. We denote by $\mathcal{Q}^{i}\left(x^{-i}\right):=\left\{x^{i} \in\right.$ $\left.\mathcal{X}^{i} \mid A x \leq b\right\}$ the set of admissible strategies for player $i$, given $x^{-i}$. To sum up, each agent aims at minimizing its cost function according to the game

$$
\mathcal{G}:=\left\{\begin{array}{rl}
\min _{x^{i} \in \mathbb{R}^{n}} & J^{i}\left(x^{i}, x^{-i}\right) \\
\text { s.t. } & x^{i} \in \mathcal{Q}^{i}\left(x^{-i}\right)
\end{array} \quad \forall i \in \mathbb{Z}[1, N],\right.
$$

where the cost functions $J^{i}$ have the aggregate structure of (1). In the following, we refer to such game as $\mathcal{G}$.

Definition 1 (Generalized Nash Equilibrium (GNE)): A point $x^{\star} \in \mathbb{R}^{N n}$ is called a generalized Nash equilibrium of the game $\mathcal{G}$ in (2) if $x^{i \star} \in \mathcal{X}^{i}, x^{\star} \in \mathcal{C}$ and $\forall i \in \mathbb{Z}[1, N]$

$$
J^{i}\left(x^{i \star}, x^{-i \star}\right) \leq J^{i}\left(x^{i}, x^{-i \star}\right), \quad \forall x^{i} \in \mathcal{Q}^{i}\left(x^{-i \star}\right) .
$$

Intuitively, a set of strategies $\left\{x^{i \star}\right\}_{i=1}^{N}$ is a GNE if it satisfies the local and global constraints and no agent can profit from unilaterally deviating from its own strategy. We denote the set of generalized Nash equilibria of the game $\mathcal{G}$ as $\operatorname{GNE}(\mathcal{G})$.
Remark 1 (Discrete-time finite-horizon dynamic games): The class of games described so far includes, for instance, the deterministic discrete-time finite-horizon dynamic games analysed in [8]. An example is given in Section VI.

\section{CONNECTION WITH VARIATIONAL INEQUALITIES}

In this section we present a reformulation of the GNE problem as an extended variational inequality, see Figure 1 for a conceptual scheme of the following results.

\section{A. GNE and variational equilibria}

The solution of GNE problems is related to the solution of certain Variational Inequalities (VI). We follow this direction to address the existence of a GNE for the game $\mathcal{G}$ in (2).

Definition 2 (Variational inequality): Consider an operator $T(\cdot): \mathbb{R}^{d} \rightarrow \mathbb{R}^{d}$ and a set $\mathcal{Y} \subseteq \mathbb{R}^{d}$. A solution to the variational inequality problem $\operatorname{VI}(\mathcal{Y}, T)$ is a vector $y^{\star} \in \mathcal{Y}$ such that $\left\langle T\left(y^{\star}\right), y-y^{\star}\right\rangle \geq 0, \forall y \in \mathcal{Y}$.

We denote the set of solutions to the $\operatorname{VI}(\mathcal{Y}, T)$ as $\operatorname{SOL}(\mathcal{Y}, T)$. Let us define the operator $F(\cdot): \mathbb{R}^{N n} \rightarrow \mathbb{R}^{N n}$ obtained by stacking together the gradient of the cost function of each player, with respect to its own strategy, that is

$$
\begin{aligned}
& F(x):=\left[\nabla_{x^{i}} J^{i}\left(x^{i}, x^{-i}\right)\right]_{i=1}^{N}=\tilde{F} x+f \\
& \tilde{F}:=\operatorname{diag}\left(Q^{i}+Q^{i \top}+\frac{C^{i \top}}{N}\right)+\frac{1}{N}\left[\begin{array}{c}
C^{1} \\
\vdots \\
C^{N}
\end{array}\right]\left(\mathbb{1}_{N}^{\top} \otimes I_{n}\right), \quad f:=\left[\begin{array}{c}
c^{1} \\
\vdots \\
c^{N}
\end{array}\right] .
\end{aligned}
$$

Moreover, let us define the sets

$$
\mathcal{X}:=\mathcal{X}^{1} \times \ldots \times \mathcal{X}^{N} \subset \mathbb{R}^{N n} \text { and } \mathcal{Q}:=\mathcal{C} \cap \mathcal{X} \subset \mathbb{R}^{N n}
$$

and introduce the following assumptions.

Assumption 1: The cost functions $J^{i}\left(x^{i}, x^{-i}\right)$ are convex in $x^{i}$ for all feasible $x^{-i}$. The local constraint sets $\mathcal{X}^{i}$ are non-empty, compact, convex and can be represented as $\mathcal{X}^{i}:=$ $\left\{x^{i} \in \mathbb{R}^{n} \mid g_{i}\left(x^{i}\right) \leq 0\right\}$ for some continuously differentiable function $g_{i}: \mathbb{R}^{n} \rightarrow \mathbb{R}^{m_{i}}, \forall i \in \mathbb{Z}[1, N]$. The global constraint set $\mathcal{Q}$ is non-empty.

Remark 2: The cost functions $J^{i}\left(x^{i}, x^{-i}\right)$ are convex in $x^{i}$ for fixed $x^{-i}$ if and only if $Q^{i}+\frac{C^{i}}{N} \succeq 0$.

Assumption 2: (Slater's constraint qualification): The sets $\mathcal{X}^{i}, i \in \mathbb{Z}[1, N]$ and $\mathcal{Q}$ satisfy the Slater's constraint qualification $[18,(5.27)]$.

The next lemma presents the connection between the $\operatorname{GNE}(\mathcal{G})$ and $\operatorname{SOL}(\mathcal{Q}, F)$.

Lemma 1: (VI reformulation [15, Theorem 5]): Suppose Assumption 1 holds. Let $x^{\star} \in \mathbb{R}^{N n}$. Then $x^{\star} \in$ $\operatorname{SOL}(\mathcal{Q}, F) \Rightarrow x^{\star} \in \operatorname{GNE}(\mathcal{G})$.

The previous lemma guarantees that $\operatorname{SOL}(\mathcal{Q}, F)$ is a subset of $\operatorname{GNE}(\mathcal{G})$. In game theory these particular equilibria are called variational equilibria, see [15]. Depending on the properties of the operator $F(\cdot)$, one can prove existence and uniqueness for $\operatorname{SOL}(\mathcal{Q}, F)$, see [13]. Here, we focus on monotone (MON) and strongly monotone (SMON) operators, as defined in the following.

Definition 3 (Monotone operator): The operator $T(\cdot)$ : $\mathbb{R}^{d} \rightarrow \mathbb{R}^{d}$ is monotone (resp. strongly monotone) if $\langle T(z)-$ $T(y), z-y\rangle \geq 0$ (resp. if $\exists c>0$ s.t. $\langle T(z)-T(y), z-y\rangle \geq$ $\left.c\|z-y\|^{2}\right), \forall z, y \in \mathbb{R}^{d}$. 
Assumption 3: The operator $F: \mathbb{R}^{N n} \rightarrow \mathbb{R}^{N n}$ introduced in (3) is strongly monotone.

Remark 3: The affine operator $F(x)$ in (3) is strongly monotone if and only if $\tilde{F}_{s}:=\frac{\tilde{F}+\tilde{F}^{\top}}{2} \succ 0$ (see e.g. [8]). Consequently, Assumption 3 implies the convexity of $J^{i}\left(x^{i}, x^{-i}\right)$ in $x^{i}$ for fixed $x^{-i}$, which is part of Assumption 1. When $C^{i}=C=C^{\top}$, the condition $\tilde{F}_{s} \succ 0$ is satisfied if $Q^{i} \succ 0$ and $C \succeq 0$ or if $Q^{i} \succeq 0$ and $C \succ 0$.

Lemma 2 (Existence \& uniqueness): Under Assumptions 1 and 3 , the $\operatorname{VI}(\mathcal{Q}, F)$ has a unique solution.

Proof: Due to Assumption 1 the set $\mathcal{Q}$ is closed and convex and $F$ is continuous. Assumption 3 guarantees strong monotonicity of $F$. The result follows from the theorem on existence and uniqueness for VIs, [13, Theorem 2.3.3].

Lemmas 1 and 2 guarantee existence and uniqueness of the variational equilibrium of the game $\mathcal{G}$, and thus ensure existence (only) of the GNE for the game $\mathcal{G}$.

\section{B. A game with $N+1$ players}

In the previous section we tackled the issue of existence of GNE for the game $\mathcal{G}$. The reformulation as a $\operatorname{VI}(\mathcal{Q}, F)$ does not readily allow us to determine the variational equilibrium with a distributed algorithm. To this end, we rewrite the $\operatorname{VI}(\mathcal{Q}, F)$ equivalently as a single shot game with $N+1$ players, but no coupling constraints

$\mathcal{G}_{\text {ext }}=\left\{\begin{array}{lrl}\min _{x^{i} \in \mathcal{X}^{i}} & J^{i}\left(x^{i}, x^{-i}\right)+\left(A_{(:, i)} x^{i}\right)^{\top} \lambda & \forall i \in \mathbb{Z}[1, N] \\ \min _{\lambda \in \mathbb{R}_{\geq 0}^{m}}-\lambda^{\top}(A x-b) & i=N+1 .\end{array}\right.$

The cost function of each player $i \in \mathbb{Z}[1, N]$ in the game $\mathcal{G}_{\text {ext }}$ is composed of two terms: the original cost function, as in $\mathcal{G}$, and an additional term that depends on the strategy $\lambda$ of player $N+1$ and on the influence of agent $i$ in the coupling constraint. The connection between the solutions of $\mathcal{G}_{\text {ext }}$ and of $\mathcal{G}$ is presented in the next lemma.

Lemma 3 (Extended game reformulation): Suppose Assumptions 1 and 2 hold. Then $\left(x^{\star}, \lambda^{\star}\right) \in \mathbb{R}^{N n} \times \mathbb{R}^{m}$ is a Nash equilibrium (NE) of the game $\mathcal{G}_{\text {ext }}$ if and only if $x^{\star} \in \mathbb{R}^{N n}$ is a solution of the $\operatorname{VI}(\mathcal{Q}, F)$ with multiplier $\lambda^{\star}$.

Proof: By Assumptions 1 and 2 the game $\mathcal{G}_{\text {ext }}$ is equivalent to the KKT systems [13]

$$
\left\{\begin{array}{rl}
\nabla_{x^{i}} J^{i}\left(x^{i}, x^{-i}\right)+A_{(:, i)}^{\top} \lambda+\nabla_{x^{i}} g_{i}\left(x^{i}\right) \mu^{i} & =0 \\
0 \leq \mu^{i} \perp-g_{i}\left(x^{i}\right) & \geq 0
\end{array} \forall i,\left\{\begin{array}{r}
-(A x-b)=\eta \\
0 \leq \eta \perp \lambda \geq 0 .
\end{array}\right.\right.
$$

Moreover $\mathcal{Q}$ satisfies the Slater's constraint qualification, by Assumption 2, and therefore, by [13, Proposition 1.3.4], $x \in$ $\operatorname{SOL}(\mathcal{Q}, F)$ if and only if there exist $\lambda,\left\{\mu^{i}\right\}_{i=1}^{N}$ such that

$$
\left\{\begin{aligned}
\nabla_{x^{i}} J^{i}\left(x^{i}, x^{-i}\right)+\nabla_{x^{i}}(A x-b) \lambda+\sum_{j=1}^{N} \nabla_{x^{i}} g_{j}\left(x^{j}\right) \mu^{j} & =0, \forall i \\
0 & \leq \mu^{i} \perp-g_{i}\left(x^{i}\right) \geq 0, \forall i \\
0 & \leq \lambda \perp-(A x-b) \geq 0 .
\end{aligned}\right.
$$

It is clear by inspection that $x$ solves (5) with multipliers $\lambda$ and $\left\{\mu^{i}\right\}_{i=1}^{N}$ if and only if $(x, \lambda)$ solves (4) with multipliers $\left\{\mu^{i}\right\}_{i=1}^{N}$ and $\eta=-(A x-b)$.

With the previous statement we moved the problem of solving the original game to the problem of solving an extended Nash equilibrium problem without coupling constraints, namely $\mathcal{G}_{\text {ext }}$. The next lemma shows that the Nash equilibria of $\mathcal{G}_{\text {ext }}$ coincide with the solutions of a second VI, defined on the extended space $[x ; \lambda]$.

Lemma 4: (Extended VI reformulation [15, Corollary 1]): Suppose Assumption 1 holds. A point $\left(x^{\star}, \lambda^{\star}\right) \in \mathbb{R}^{N n} \times \mathbb{R}^{m}$ is a Nash equilibrium for $\mathcal{G}_{\text {ext }}$ if and only if it is a solution of the $\operatorname{VI}(\mathcal{Y}, T)$, with $\mathcal{Y}:=\mathcal{X} \times \mathbb{R}_{\geq 0}^{m}$ and affine operator

$$
\begin{aligned}
T(x, \lambda): & =\left[\begin{array}{c}
{\left[\nabla_{x^{i}} J^{i}\left(x^{i}, x^{-i}\right)+\nabla_{x^{i}}\left(A_{(:, i)} x^{i}\right) \lambda\right]_{i=1}^{N}} \\
-(A x-b)
\end{array}\right] \\
& =\left[\begin{array}{cc}
\tilde{F} & A^{\top} \\
-A & 0
\end{array}\right]\left[\begin{array}{l}
x \\
\lambda
\end{array}\right]+\left[\begin{array}{l}
f \\
b
\end{array}\right]=: \tilde{T} y+t .
\end{aligned}
$$

\section{A unifying picture}

We recap the previous results with the following theorem.

Theorem 1: Under Assumptions 1, 2 and 3 the game $\mathcal{G}$ in (2) has a unique variational equilibrium $x^{\star}$. If $(\bar{x} ; \bar{\lambda})$ is a solution of $\operatorname{VI}(\mathcal{Y}, T)$, as defined in Lemma 4 , then $\bar{x}=x^{\star}$.

Proof: This result follows from Lemmas 1, 2, 3 and 4. See Figure 1 for a conceptual scheme.

Under Assumption 3 the operator $T(x, \lambda)$ is MON, as proven in the next lemma, therefore the solutions of $\operatorname{VI}(\mathcal{Y}, T)$ can be found using algorithms for VIs with MON operator.

Lemma 5: Under Assumption 3, $T(x, \lambda)$ defined in (6) is MON and Lipschitz.

Proof: $T(y):=\tilde{T} y+t$ is an affine operator hence it is Lipschitz with Lipschitz constant $L_{T}:=\|\tilde{T}\|$. Moreover, it is MON if and only if $\tilde{T}_{s}=\left[\begin{array}{cc}\tilde{F}_{s} & 0 \\ 0 & 0\end{array}\right] \succeq 0$, which holds true thanks to Assumption 3.

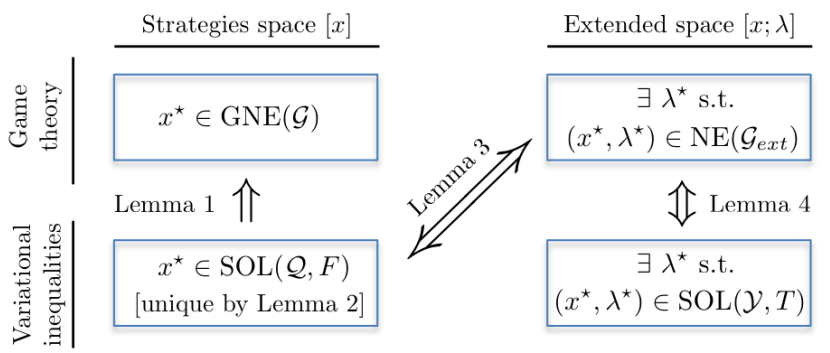

Fig. 1: Conceptual scheme for Theorem 1.

\section{TOWARDS A Distributed CALCULATION OF GNE}

The main advantage of the reformulation given in Theorem 1 is that the set $\mathcal{Y}$ decouples in the Cartesian product of local constraint sets, so that projection operations can be computed locally by each agent. Additionally, for each agent the gradients of the first and second term of the cost function in $\mathcal{G}_{\text {ext }}$ depend only on the local strategy $x^{i}$ and on the aggregate quantity $\sigma(x)$, that is,

$$
\begin{aligned}
\nabla_{x^{i}} J^{i}\left(x^{i}, x^{-i}\right) & =\left(Q^{i}+Q^{i \top}+\frac{C^{i^{\top}}}{N}\right) x^{i}+C^{i} \sigma(x)+c^{i} \\
& =: F^{i}\left(x^{i}, \sigma(x)\right) \in \mathbb{R}^{n} \\
\nabla_{x^{i}}\left(A_{(:, i)} x^{i}\right) & =A_{(:, i)}^{\top} \in \mathbb{R}^{n \times m} .
\end{aligned}
$$

With this notation the operator $T(x, \lambda)$ can be rewritten as

$$
T(x, \lambda):=\left[\begin{array}{c}
{\left[F^{i}\left(x^{i}, \sigma(x)\right)+A_{(:, i)}^{\top} \lambda\right]_{i=1}^{N}} \\
-(A x-b)
\end{array}\right] .
$$


The fact that both gradient and projection operations can be computed locally by the agents is the fundamental feature that allows us to present a decentralized scheme for the solution of $\operatorname{VI}(\mathcal{Y}, T)$, thus guaranteeing decentralized convergence to the variational equilibrium of $\mathcal{G}$.

\section{A. The asymmetric projection algorithm}

In this section we propose a new algorithm to find a solution $(\bar{x}, \bar{\lambda})$ of the $\operatorname{VI}(\mathcal{Y}, T)$. The proposed algorithm alternates updates in the primal $(x)$ and dual variables $(\lambda)$. We assume the presence of a central operator that broadcasts a tentative price $\lambda_{(k)}$ at every step $k$ of the algorithm. Based on this price and on the aggregate of the strategies at the previous step, each agent locally computes $x_{(k+1)}^{i}$. Based on the global constraint violation, the central operator updates the price to $\lambda_{(k+1)}$. The proposed scheme differs from the

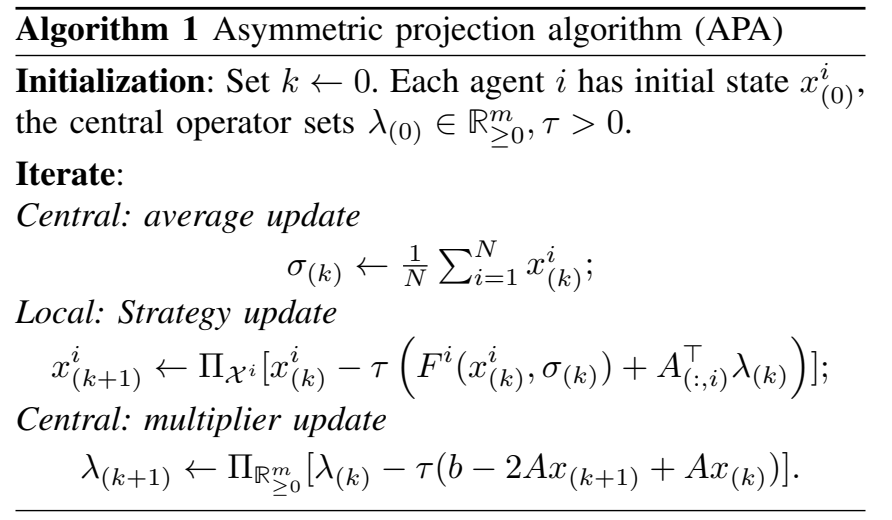

standard gradient projection algorithm, which is not guaranteed to converge in the presence of coupling constraints [13, Algorithm 12.1.1], since the $\lambda$-update depends not only on $A x_{(k)}$ but also on $A x_{(k+1)}$. Algorithm 1 belongs to the class of Asymmetric Projection Algorithms (APA) [13, Algorithm 12.5.1]. The convergence properties of these algorithms have been previously characterized in the literature for the case of a $\operatorname{VI}(K, M)$ with convex domain $K$ and affine operator $M(y)=\left[\begin{array}{rr}P & R \\ -R^{\top} & Q\end{array}\right] y+\left[\begin{array}{l}p \\ q\end{array}\right]$, where $P=P^{\top} \succeq 0, Q=Q^{\top} \succeq$ 0 [13, Proposition 12.5 .3 (b)]. The operator $T(y)$ that we are considering, however, does not belong to this class since $\tilde{T}=\left[\begin{array}{cc}\tilde{F} & A^{\top} \\ -A & 0\end{array}\right]$, but in general $\tilde{F} \neq \tilde{F}^{\top}$. We show in the next theorem that the condition $\tilde{F} \succ 0$ guarantees convergence of Algorithm 1 for this new class of affine VIs. We note that the missing assumption $\tilde{F}=\tilde{F}^{\top}$, even if seemingly mild, greatly simplifies the analysis because it turns the game in (2) into a potential game, as further discussed in Section V.

Theorem 2: Suppose Assumptions 1, 2 and 3 hold. Let $y_{(k)}:=\left[x_{(k)} ; \lambda_{(k)}\right]$ be the extended state of the population at iteration $k$ obtained by Algorithm 1. Let

$\kappa_{F}:=\frac{\sigma_{\max }^{2}(\tilde{F})}{\sigma_{\min }\left(\tilde{F}_{s}\right)}, \quad 0<\tau<\left(\frac{\kappa_{F}+\sqrt{\kappa_{F}^{2}+4\left\|A^{\top} A\right\|}}{2}\right)^{-1}$.

Then $y_{(k)} \stackrel{k \rightarrow \infty}{\longrightarrow} \bar{y}:=[\bar{x} ; \bar{\lambda}]$ where $\bar{y}$ is a solution of the $\operatorname{VI}(\mathcal{Y}, T)$, with $T(x, \lambda)$ defined in (6), and hence $\bar{x}$ is the variational equilibrium of the game $\mathcal{G}$ in (2).
Due to space limitation, the proof of Theorem 2 and the proof of the next proposition are in [19].

Proposition 1: (Rate of convergence) Define $B=$ $D_{s}^{-1 / 2}\left(D-D_{s}\right) D_{s}^{-1 / 2}$ with $D:=\left[\begin{array}{cc}\frac{1}{\tau} I_{N n} & 0 \\ -2 A & \frac{1}{\tau} I_{m}\end{array}\right]$ and assume that $\|B\|<1$ and that $\mathcal{X}$ is a polyhedron. Under the assumptions of Theorem 2, Algorithm 1 converges $R$-linearly:

$$
0<\lim \sup _{k \rightarrow \infty}\left(\left\|y_{(k)}-\bar{y}\right\|\right)^{1 / k}<1 .
$$

Remark 4: The condition $\|B\|<1$ can be satisfied by choosing $\tau$ sufficiently small. In fact $D-D_{s}$ does not depend on $\tau$ and $D_{s}^{-1 / 2}=O\left(\tau^{1 / 2}\right)$ [13, Proposition 12.5.4].

Finally, in the interest of completeness, we note that since the operator $T$ is MON, there are other algorithms available in the literature to find a solution of $\operatorname{VI}(\mathcal{Y}, T)$, e.g. the extragradient method [13, Algorithm 12.1.9]. A comparison between these two algorithms is given in Section VI.

\section{Connection With potential games}

The objective of this section is to show how the previous analysis simplifies under the symmetry assumption $\tilde{F}=\tilde{F}^{\top}$ and to provide a link between game theory and single objective optimization. To this end, we start by recalling the definition of generalized potential games.

Definition 4 (Generalized potential game [16]): $\mathcal{G}$ is said to be a generalized potential game if there exists a function $P\left(x^{1}, \ldots, x^{N}\right): \mathbb{R}^{N n} \rightarrow \mathbb{R}$, such that $\forall i \in \mathbb{Z}[1, N]$ and $\forall x^{-i}$ such that $\mathcal{Q}^{i}\left(x^{-i}\right)$ is non empty, it holds $\forall y^{i}, z^{i} \in \mathcal{Q}^{i}\left(x^{-i}\right)$

$$
J^{i}\left(y^{i}, x^{-i}\right)-J^{i}\left(z^{i}, x^{-i}\right)=P\left(y^{i}, x^{-i}\right)-P\left(z^{i}, x^{-i}\right) .
$$

In words, a game is potential if the difference that each player encounters by unilaterally changing its strategy can be captured by the difference of a single scalar function $P(\cdot)$, called the potential. When the cost function of each player depends only on his decision variable, the game is potential with $P(x)$ the sum of all the players costs, see [20], [21]. Under the following assumption it can be shown that the game in (2) is a generalized potential game.

Assumption 4: For each agent $i, C^{i}=C=C^{\top}$.

Proposition 2: Under Assumption 4, $\mathcal{G}$ is potential with

$$
P(x)=\frac{1}{2} x^{\top} \tilde{F} x+f^{\top} x,
$$

where $\tilde{F}$ and $f$ are as in (3).

Proof: For each agent $i$ and fixed strategies $x^{-i}$ it holds

$$
\nabla_{x^{i}} P\left(x^{i}, x^{-i}\right)=\nabla_{x^{i}} J^{i}\left(x^{i}, x^{-i}\right)=F^{i}\left(x^{i}, \sigma(x)\right), \forall x^{i} .
$$

The fundamental theorem of calculus implies that, for fixed $x^{-i}$, the difference in cost that agent $i$ encounters playing $z^{i}$ instead of $y^{i}$ is the same in the individual cost $J^{i}\left(y^{i}, x^{-i}\right)-$ $J^{i}\left(z^{i}, x^{-i}\right)$ as in the potential $P\left(y^{i}, x^{-i}\right)-P\left(z^{i}, x^{-i}\right)$.

One of the main features of potential games is that their variational equilibria are related to the minima of the potential function, thus simplifying the analysis.

Theorem 3 (Optimization problem reformulation): Under Assumptions 1, 3 and $4, x^{\star}$ is the variational equilibrium of $\mathcal{G}$ if and only if it is the unique minimizer of $P(x)$ in (7) over the set $\mathcal{Q}=\mathcal{X} \cap \mathcal{C}$.

Proof: Lemmas 1 and 2 ensure that the variational equilibrium exists and is the unique solution to $\operatorname{VI}(Q, F)$. Assumptions 1 and 3 imply that $P(x)$ is strongly convex and 
that $\mathcal{Q}$ is convex and compact. This guarantees uniqueness of the minimizer of $P(x)$ in $\mathcal{Q}$. The point $x$ is the minimizer of $P(x)$ on $\mathcal{Q}$ if and only if $\left\langle\nabla_{x} P(x), y-x\right\rangle \geq 0$ for all $y \in Q[18,(4.21)]$. By (3) and (8), the latter coincides with $\langle F(x), y-x\rangle \geq 0$ for all $y \in Q$, i.e. $x \in \operatorname{SOL}(\mathcal{Q}, F)$.

Remark 5: The previous theorem clarifies how a GNE of $\mathcal{G}$ can be found solving a single objective optimization problem. We direct the reader to [22] for a comprehensive review of distributed optimization methods.

\section{Charging a Fleet of PEV}

Inspired by [17], we model the problem of charging a large population of Plug-in Electric Vehicles (PEVs) as a game with coupling constraints. The population is composed by $N$ electric vehicles; each of them fitted with a battery whose state of charge at time $t$ is described by $s_{t}^{i}$ and takes values in $[0,1]$. The time evolution of $s_{t}^{i}$ is specified by the discretetime system $s_{t+1}^{i}=s_{t}^{i}+b^{i} x_{t}^{i}, t=1, \ldots, n$, where $x_{t}^{i}$ is the charging control for vehicle $i$ at time $t$ and $b^{i}$ is the charging efficiency. We assume that the charging control cannot take negative values and its magnitude is bounded from above. The final state of charge is constrained to $s_{n+1}^{i}=\eta^{i}$, where $\eta^{i}$ is decided by each user. We denote with $P^{i} \subset \mathbb{Z}[1, n]$ the set of intervals in which vehicle $i$ cannot charge (for instance, because the owner is driving the car). Denoting $x^{i}=\left\{x_{1}^{i}, \ldots, x_{n}^{i}\right\} \in \mathbb{R}^{n}$, these three constraints can be expressed together as

$$
x^{i} \in \mathcal{X}^{i}:=\left\{\begin{array}{l|l}
x^{i} \in \mathbb{R}^{n} \mid \begin{array}{l}
0 \leq x_{t}^{i} \leq \bar{x}^{i} \\
x_{t}^{i}=0 \\
\sum_{t=1}^{n} x_{t}^{i}=\theta^{i}
\end{array}
\end{array}\right\}
$$

where $\theta^{i}:=\left(b^{i}\right)^{-1}\left(\eta^{i}-s_{1}^{i}\right)$, with $s_{1}^{i}$ being the initial state of charge. Note that $\mathcal{X}^{i}$ is compact and convex for all $i$.

In addition to being subject to the individual constraints $\mathcal{X}^{i}$, the vehicles are subject to the global coupling constraint

$$
\frac{1}{N} \sum_{i=1}^{N} x_{t}^{i} \leq K_{t}, \quad \forall t \in \mathbb{Z}[1, n],
$$

expressing the fact that the grid cannot deliver more than $K_{t}$ normalized units of power to the PEVs at any time $t$. In compact form (9) reads as $\left(\mathbb{1}_{N} \otimes I_{n}\right) x \leq N K$, where $K=\left[K_{1}, \ldots, K_{n}\right]$. We assume $\mathcal{Q}=\mathcal{C} \cap \mathcal{X}$ non empty. Each vehicle seeks a strategy $x^{i}$ that minimizes the cost

$$
J^{i}\left(x^{i}, x^{-i}\right):=\sum_{t=1}^{n} p^{i}\left(\frac{d_{t}+\sigma_{t}}{\kappa}\right) x_{t}^{i},
$$

where $d_{t}$ and $\sigma_{t}$ are the non-PEV and PEV demand at time $t$ divided by $N$, respectively, and $\kappa$ is the total production capacity divided by $N$; the price $p^{i}$ is then a function of $\left(d_{t}+\right.$ $\left.\sigma_{t}\right) / \kappa$, that is, of the ratio between total consumption and total capacity. The cost function (10) represents the energy bill for agent $i$ over the interval $\mathbb{Z}[1, n]$; that is, for each time interval $t$, agent $i$ buys $x_{t}^{i}$ units of electric power at the unit price $p^{i}\left(\frac{d_{t}+\sigma_{t}}{\kappa}\right)$. We assume in the following that both $d_{t}$ and $\kappa$ are known to each PEV, and that the unit price is an affine increasing function of its argument, i.e. $p^{i}(z)=\alpha^{i} z+\beta^{i}$ with $\alpha^{i}, \beta^{i}>0$. Different price functions $p^{i}$ can be due to the fact that the PEVs participate to pricing schemes of different utilities. The cost (10) then becomes $J^{i}\left(x^{i}, x^{-i}\right)=\left(C^{i} \sigma+c^{i}\right)^{\top} x^{i}$, with $C^{i}:=\left(\alpha^{i} / \kappa\right) I_{n} \succ 0$ and $c_{t}^{i}:=\alpha^{i} d_{t} / \kappa+\beta^{i}>0$. We now define $\mathcal{G}_{\mathrm{PEV}}$ as

$\mathcal{G}_{\mathrm{PEV}}:=\left\{\begin{array}{l}\min _{x^{i}} J^{i}\left(x^{i}, x^{-i}\right) \\ \text { s.t. } x^{i} \in \mathcal{X}^{i},\left(\mathbb{1}_{N} \otimes I_{n}\right) x \leq N K .\end{array} \forall i \in \mathbb{Z}[1, N]\right.$

The PEVs charging problem has been formulated as a game also in [17] and [8]. The main differences between those works and the PEVs game in (11) are: (i) we guarantee convergence to a Nash equilibrium for any finite population size; (ii) the cost function (10) does not include an artificial quadratic term, required in [17] and [8]; (iii) we introduce the coupling constraints (9). The first two novelties have already been presented in [6]. Point (iii) has not yet been addressed in the PEV literature, to the best of our knowledge.

\section{A. Numerical analysis}

The numerical study is conducted on a heterogeneous population, where the agents differ in $\theta^{i}, \bar{x}^{i}$, randomly chosen according to $\mathcal{U}[0.5,1.5]$ and $\mathcal{U}[1,5]$, respectively; they also differ in $P^{i} \subset \mathbb{Z}[1, n]$ (connected interval, with left and right endpoints uniformly randomly chosen). Moreover $\alpha^{i}=\alpha=1$ and $\beta^{i}=\beta=1 \forall i \in \mathbb{Z}[1, n]$. The demand $d_{t}$ is taken as the typical (non-PEV) base demand over a summer day in the United States [17, Figure 1]; $\kappa=1 \mathrm{~kW}$, and the upper bound $K_{t}=0.55 \mathrm{~kW}$ is chosen such that the coupling constraint (9) is active in the middle of the night. The GNEP (11) is a particular case of (2), hence the analysis conducted in Sections III and IV applies. Assumption 1 holds because $\alpha>0, \mathcal{X}^{i}$ are convex polytopes and we assumed $\mathcal{Q}$ non empty. Assumption 2 holds because all the constraints are affine and non empty. Finally, $\alpha>0$ guarantees that Assumption 3 is satisfied. Numerical results are in Figure 2.

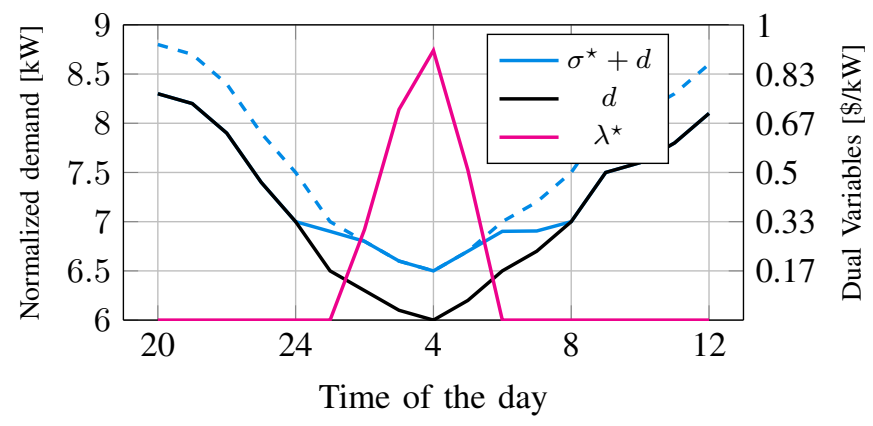

Fig. 2: Aggregate PEV demand $\sigma^{\star}$ and dual variables $\lambda^{\star}$ for $N=$ 100 , subject to $\sigma^{\star} \leq 0.55 \mathrm{~kW}$. The region below the dashed line corresponds to $\sigma^{\star}+d \leq 0.55 \mathrm{~kW}+d$.

Along with the APA (Algorithm 1), we implemented the extragradient algorithm [13, Algorithm 12.1.9], setting as stopping criterion $\left\|\left(x_{(k+1)}, \lambda_{(k+1)}\right)-\left(x_{(k)}, \lambda_{(k)}\right)\right\|_{\infty} \leq \epsilon_{\text {toll }}$. Figure 3 (top) shows that Algorithm 1 outperforms the extragradient method, requiring less computations for large populations.

\section{B. GNE vs social optimum}

We also compare numerically the variational GNE of (11) with the optimal solution $x_{S}^{\star}$ of the centralized social welfare problem, which is defined as follows

$$
x_{S}^{\star}:=\arg \min _{x \in \mathbb{R}^{N n}} S(x) \quad \text { s.t. } x^{i} \in \mathcal{X}^{i}, \quad\left(\mathbb{1}_{N}^{\top} \otimes I_{N}\right) x \leq K,
$$



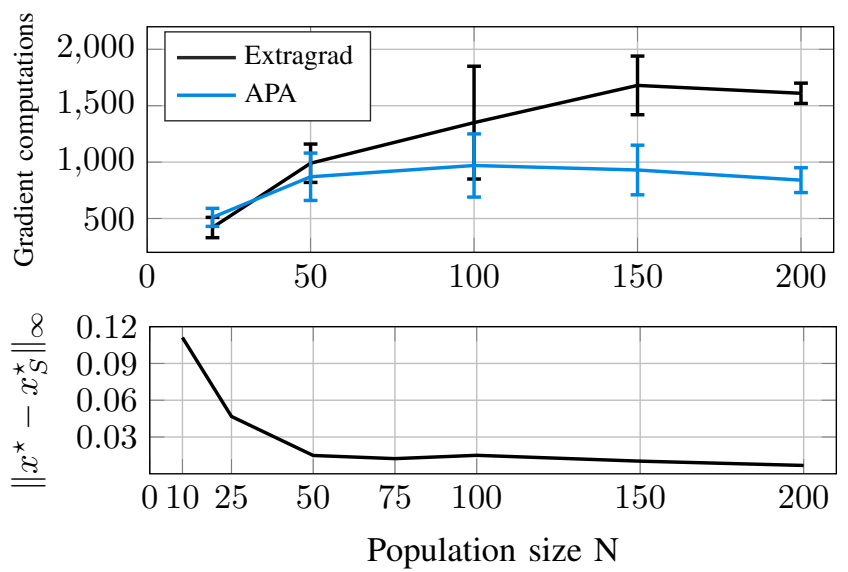

Fig. 3: Number of gradient computations for convergence (with $\epsilon_{\text {toll }}=10^{-4}$ ); mean and standard deviation relative to 10 repetitions are reported (top). Difference between the variational GNE and the social optimum (bottom).

where $S(x)=\sum_{t=1}^{n}\left(\alpha \frac{d_{t}+\sigma_{t}}{\kappa}+\beta\right)\left(d_{t}+\sigma_{t}\right)$ represents the electricity cost for the entire city, both for PEVs and for nonPEVs. The work [23] shows that in the case of homogeneous population (i.e., when $\mathcal{X}^{i}$ is the same for all $i$ ), $x_{S}^{\star}$ tends to $x^{\star}$, as the population size $N$ goes to infinity. The numerical analysis of Figure 3 (bottom) suggests that this is the case also for heterogeneous players with coupling constraints.

\section{Local coupling constraints}

Instead of the coupling constraint (9), we consider here $m=H \cdot n$ local coupling constraints of the form $\sum_{j \in \mathcal{N}_{h}} x_{t}^{j} \leq K^{h}, \forall t \in \mathbb{Z}[1, n], \quad \forall h \in \mathbb{Z}[1, H]$ where $\mathcal{N}_{h} \subset \mathbb{Z}[1, N]$ represents the subset of agents connected to the same transformer $h$, which cannot provide more than $K^{h}$ units of power at any time $t$. Figure 4 shows how such coupling constraint forces a coordination between different players: player 2 charges its PEV in the first part of the night, and only when he stops, player 1 starts to charge.

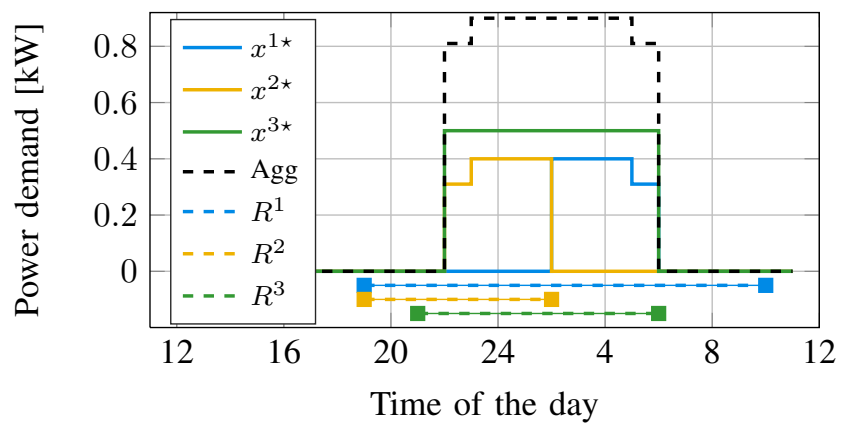

Fig. 4: GNE strategies and their aggregate $x^{1 \star}+x^{2 \star}+x^{3 \star}$, together with the charging intervals $R^{1}, R^{2}, R^{3}$. The local coupling constraint is $x_{t}^{1 \star}+x_{t}^{2 \star}+x_{t}^{3 \star} \leq 0.9 \mathrm{~kW}$.

\section{CONCLUSIONS}

We analysed quadratic aggregative games subject to both private and coupling constraints. We obtained sufficient conditions for the existence of a generalized Nash equilibrium and proposed a decentralized algorithm that converges to it. Our theoretical results allow us to extend previous literature results on the charging problem of a fleet of PEVs. When more than one GNE exists, one could study algorithms that can steer the population to the most favourable GNE [24].

\section{REFERENCES}

[1] T. Başar and G. Olsder, Dynamic noncooperative game theory. Society for Industrial and Applied Mathematics, 1999.

[2] R. W. Rosenthal, "A class of games possessing pure-strategy Nash equilibria," International Journal of Game Theory, vol. 2, no. 1, pp. 65-67, 1973.

[3] T. Alpcan, T. Başar, R. Srikant, and E. Altman, "CDMA uplink power control as a noncooperative game," Wireless Networks, vol. 8, pp. 659670, 2002.

[4] H. Chen, Y. Li, R. H. Louie, and B. Vucetic, "Autonomous demand side management based on energy consumption scheduling and instantaneous load billing: An aggregative game approach," IEEE Trans. on Smart Grid, vol. 5, no. 4, pp. 1744-1754, 2014.

[5] M. K. Jensen, "Aggregative games and best-reply potentials," Economic theory, vol. 43, no. 1, pp. 45-66, 2010.

[6] D. Paccagnan, M. Kamgarpour, and J. Lygeros, "On Aggregative and Mean Field Games with Applications to Electricity Markets," in Proceedings of the IEEE ECC, Jun. 2016.

[7] J. Koshal, A. Nedic, and U. V. Shanbhag, "A gossip algorithm for aggregative games on graphs," in Proceedings of the IEEE CDC, 2012, pp. $4840-4845$.

[8] S. Grammatico, F. Parise, M. Colombino, and J. Lygeros, "Decentralized convergence to Nash equilibria in constrained deterministic mean field control," IEEE Trans. on Automatic Control (in press).

[9] F. Parise, S. Grammatico, B. Gentile, and J. Lygeros, "Network aggregative games and distributed mean field control via consensus theory," ArXiv. Available at http://arxiv.org/pdf/1506.07719.pdf, 2015.

[10] M. Huang, P. E. Caines, and R. P. Malhamé, "Large-population costcoupled LQG problems with nonuniform agents: Individual-mass behavior and decentralized $\varepsilon$-Nash equilibria," IEEE Trans. on Automatic Control, vol. 52, no. 9, pp. 1560-1571, 2007.

[11] J.-M. Lasry and P.-L. Lions, "Mean field games," Japanese Journal of Mathematics, vol. 2, no. 1, pp. 229-260, 2007.

[12] J. B. Rosen, "Existence and uniqueness of equilibrium points for concave N-person games," Econometrica: Journal of the Econometric Society, pp. 520-534, 1965.

[13] F. Facchinei and J.-S. Pang, Finite-dimensional variational inequalities and complementarity problems. Springer, 2007.

[14] M. Zhua and E. Frazzoli, "Distributed robust adaptive equilibrium computation for generalized convex games," Automatica, vol. 63, pp. 82-91, 2016.

[15] F. Facchinei and C. Kanzow, "Generalized Nash equilibrium problems," 4OR, vol. 5, no. 3, pp. 173-210, 2007.

[16] F. Facchinei, V. Piccialli, and M. Sciandrone, "Decomposition algorithms for generalized potential games," Computational Optimization and Applications, vol. 50, no. 2, pp. 237-262, 2011.

[17] Z. Ma, D. S. Callaway, and I. A. Hiskens, "Decentralized charging control of large populations of plug-in electric vehicles," IEEE Trans. on Control Systems Technology, vol. 21, no. 1, pp. 67-78, 2013.

[18] S. Boyd and L. Vandenberghe, Convex optimization. Cambridge university press, 2004.

[19] D. Paccagnan, B. Gentile, F. Parise, M. Kamgarpour, and J. Lygeros, "Distributed computation of generalized Nash equilibria in quadratic aggregative games with affine coupling constraints," Tech. Rep., 2016, http://control.ee.ethz.ch/index.cgi? page $=$ publications; action=details;id $=5485$.

[20] J. Koshal, A. Nedic, and U. V. Shanbhag, "Multiuser optimization: distributed algorithms and error analysis," SIAM Journal on Optimization, vol. 21, no. 3, pp. 1046-1081, 2011.

[21] S. H. Low and D. E. Lapsley, "Optimization flow control, I: basic algorithm and convergence," IEEE/ACM Transactions on Networking, vol. 7, no. 6, pp. 861-874, 1999.

[22] D. P. Bertsekas and J. N. Tsitsiklis, Parallel and distributed computation: numerical methods. Prentice-Hall, Inc., 1989.

[23] M. G. Vaya, S. Grammatico, G. Andersson, and J. Lygeros, "On the price of being selfish in large populations of plug-in electric vehicles," in Proceedings of the IEEE CDC, 2015, pp. 6542-6547.

[24] N. Li and J. R. Marden, "Designing games for distributed optimization," IEEE Journal of Selected Topics in Signal Processing, vol. 7, no. 2, pp. 230-242, 2013. 